\title{
L'ENFANCE DU MONDE. PREMIERS CONTACTS ENTRE FRANÇAIS ET TUPINAMBAS DANS LE BRÉSIL DU XVIE SIÈCLE
}

Laurent Olivier ${ }^{1}$

\section{Résumé}

Ce texte discute des premiers contacts entre Français et Tupinambas au Brésil du XVIe siècle, envisageant surtout les réflexions de Jean de Léry à propos de la notion de "sauvage" et de rencontres et d'échanges culturels.

\section{Mots-clés}

Brésil/France, Tupinambas, «Sauvages », rencontres/échanges culturels

\section{Resumo}

Este texto trata dos primeiros contatos entre franceses e tupinambás no Brasil do século XVI explorando principalmente as reflexões de Jean de Léry sobre a noção de "selvagem" e de encontro e trocas culturais.

\section{Palavras-chave}

Brasil/França, Tupinambás, "selvagens", encontro/trocas culturais

\footnotetext{
${ }^{1}$ Conservateur en chef, Musée d'Archéologie Nationale, Saint-Germain-en-Laye, France.

E-mail: laurent.olivier@culture.gouv.fr
} 
La terre n'est d'abord qu'une senteur indécise, qui vient par moments $\mathrm{s}^{\prime}$ insinuer dans la rumeur de la mer. Ce sont des lambeaux d'un parfum lointain de forêt, de fruits mûrs et d'épais humus. Un matin, soudain, elle est là, dont la masse grise des montagnes domine à l'horizon la surface de la mer. On la pressent immense, bien trop grande pour nous. Pendant plusieurs jours et plusieurs nuits, le navire approche d'une interminable succession de côtes désertes, où nul feu ne brille dans l'obscurité. De plus près, la terre paraît gardée par d'énormes rochers émergeant de la surface de la forêt, qui, par endroits, semble tomber directement dans la mer. À perte de vue, on ne distingue pas la moindre trace d'habitation. De temps en temps, le silence navrant de la terre est percé par l'écho du cri des singes et des perroquets. Porté par la masse énorme du bateau, on s'avance au-devant d'un pays vierge de toute marque humaine, où abondent les oiseaux et les animaux, comme aux premiers temps du monde.

Ils sont là, pourtant. La forêt grouille de milliers de sauvages nus, de terribles populations cannibales, sur lesquelles circulent les histoires les plus horribles. C'est le Brésil. Notre Brésil perdu, le Brésil des origines. Les huguenots français y ont fondé une minuscule colonie en 1555, sous l'autorité de Nicolas Durand de Villegagnon, vice-amiral de Bretagne. Ils se sont installés à l'embouchure du fleuve Guanabara, sur une île qui sera connue sous le nom d'île-aux-Français. Deux cents émigrants sont rassemblés dans un fortin baptisé Fort Coligny, en hommage à leur protecteur, chef du parti protestant de France. La petite colonie de la «France antarctique » ne connaîtra qu'une brève existence, avant qu'elle ne soit détruite en 1560 par les Portugais².

Cette implantation éphémère nous aura confronté à l'étrange et au merveilleux, à la beauté et à la terreur. Elle nous a fait connaitre pour la première fois les "Sauvages américains", en l'occurrence les Toüoupinambaoults ou Tupinambas, une fraction des populations Tupi de la Baie de Rio de Janeiro, qui s'éteindra au XVIIe siècle. Surtout, l'expérience de l'altérité sauvage que nous aura offert la courte présence française au Brésil aura fait ressurgir une Antiquité que l'on pouvait croire disparue à jamais. Nous qui n'avions jamais connu que les peuples du Vieux Monde - étrangers certes, mais néanmoins voisins de ce que nous étions - nous avons soudainement été confrontés à la dissemblance absolue, comme l'avaient été auparavant les Grecs et les Romains lorsqu'ils s'étaient avancés aux confins du monde connu. L'Antiquité est revenue sous sa forme la plus inattendue, la moins visible: comme une expérience. L'expérience de l'Autre, de sa proximité et de son étrangeté totales. Et, avec elle, la découverte d'une

\footnotetext{
${ }^{2}$ Rasé par les Portugais, Fort Coligny peut être considéré comme le premier établissement européen permanent à l'origine de la fondation ultérieure de la ville de Rio de Janeiro.
} 
conscience, encore embryonnaire, de la fin d'un monde, somptueux et fragile, dont nous provoquions directement l'extinction.

\section{Rencontre avec les « Sauvages »}

De cette histoire sans lendemain, il ne serait rien resté, ou si peu, si un jeune cordonnier de Bourgogne converti au Protestantisme n'avait fait le voyage du Brésil en 1557. Envoyé par Calvin à Fort Coligny pour y rejoindre la petite communauté huguenote, Jean de Léry rapporte de son séjour en "France antarctique » le récit de son expérience au contact des Tupinambas, partagé entre son admiration pour la générosité des «Sauvages » et sa répulsion vis-àvis de leurs pratiques païennes ${ }^{3}$. Admiré de Claude Lévi-Strauss, le livre de Léry est le premier ouvrage d'ethnographie amérindienne du Brésil. C'est surtout le témoignage d'un homme ordinaire, soudainement projeté dans l'univers singulier des "Sauvages de l'Amérique», dont il découvre, à son grand étonnement, les modes de vie et les codes de conduite.

On ne connaît alors le Nouveau Monde que par ouïe dire, par les histoires de ceux qui en sont revenus. On raconte que l'Amérique est peuplée de géants hauts de plus de trois mètres, avec deux rangées de dents en haut et en bas. Certains disent $\mathrm{qu}^{\prime} \mathrm{y}$ habite un peuple de nains dont la vie est très courte, et qui meurent avant que nous-mêmes n'ayons atteint l'âge adulte. D'autres assurent qu'on y a vu des hommes-singes, pourvu d'une queue velue, et qu'il existe là-bas des hommes couverts d'un pelage aussi épais que celui des ours. Il est impossible de démêler la fiction de la réalité. Les hommes les plus respectables colportent les histoires les plus invraisemblables, comme celle des bêtes à tête d'enfant qui ne se nourrissent que de vent, dont le grand savant André Thevet, cosmographe du roi François Ier, assure avoir tenu entre les mains un exemplaire pris au Brésil (fig. 1) $)^{4}$. Du coup, on n'est pas sûr que certains prodiges ne puissent finalement être possibles aussi, comme celui des hommes dont la tête est dans la poitrine, ou encore celui des êtres qui se réduisent à un pied énorme, dont ils se servent pour s'abriter du soleil brûlant.

\footnotetext{
3 Jean de Léry, Histoire d'un voyage faict en la terre du Brésil, autrement dite Amérique. Genève, Antoine Chuppin, 1578. Particulièrement populaire, l'ouvrage connaîtra cinq éditions, jusqu'en 1611. Publié vingt ans après le voyage de 1557, le livre de Léry est une réponse à l'ouvrage qu'avait fait paraître en 1575 le catholique André Thevet. Dans ses Singularités de la France antarctique, Thevet attribuait aux protestants la responsabilité de l'échec de l'établissement français de Fort Coligny.

${ }^{4}$ Il s'agit de l'Haüt, ou Haüthi. André Thevet, Le Brésil d'André Thevet. Singularités de la France antarctique (1557). Paris 1997, éditions Chandeigne, p. 199-200.
} 


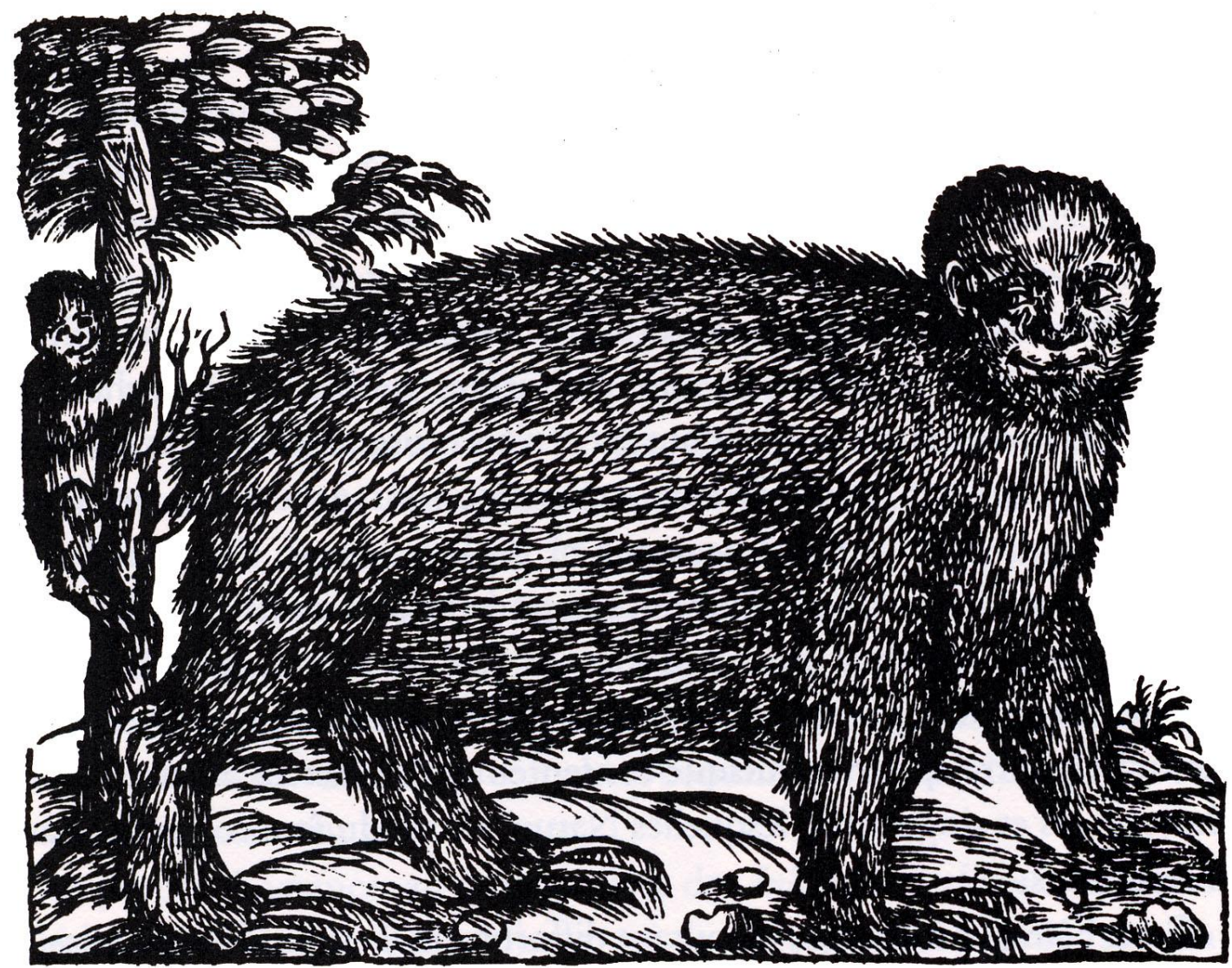

Fig. 1. Haut ou Hauthi - André Thévet. Les singularitez de la France Antarctique, 1557.

Ces chimères sont le produit d'un enfantement particulier : elles sont issues de la rencontre des vieilles fables européennes - avant de les voir aux Amériques, on plaçait la plupart de ces prodiges en Inde, et avant encore sur les rivages lointains du pays des Scythes - et de l'identité insaisissable du monde indigène. L'étrangeté du Nouveau Monde vient en quelque sorte au-devant des Européens avant même qu'ils ne l'aient encore réellement abordée. C'est pourquoi on prête considération à ces fictions: elles arrivent comme des signes, qui parlent dans la langue de l'inconnu. Elles réhabilitent désormais les histoires, que l'on croyait volontiers mensongères, qu'avaient rapportées les Anciens de leurs voyages au bout du monde, tel Pythéas, l'inventeur d'Ultima Thulé, la terre extrême du monde des glaces ${ }^{5}$. Revenu de son voyage, Léry, fera ainsi amende honorable: «j'avoue, écrit-il, que je n'aurai point de honte de confesser que depuis que j'ai été en ce pays d'Amérique (...) sans approuver les fables qui se lisent ès livres de plusieurs, lesquels se fiant aux rapports qu'on leur a faits ou, autrement, ont écrit choses du tout fausses, je me suis rétracté de l'opinion que j'ai autrefois eue de Pline, et de quelques autres

\footnotetext{
5 Le géographe grec Strabon fera ainsi du navigateur Pythéas «le plus menteur des hommes » (Strabon, Géographie, IV, 3, 5).
} 
décrivant les pays étranges, parce que j'ai vu des choses aussi bizarres et prodigieuses qu'aucunes qu' on a tenues incroyables dont ils font mention. »6

Ce n'est pas tant de trouver un pays complètement différent du leur qui étonne les premiers Européens accostant au Brésil : c'est d'y rencontrer des êtres humains. En face d'eux, les foules d'êtres nus qui se massent à leur rencontre ne croient pas non plus possible que de simples hommes puissent arriver de l'endroit par où ils sont venus, là où, à l'horizon, le ciel touche la mer. La rencontre avec l'humanité du Nouveau Monde procède d'un énorme malentendu. Chacun voit l'autre non comme il est - ou tel qu'il se considère être - mais comme ce que celui-là représente pour soi. Et chacun ignore ce que lui-même représente pour cet autre, qu'il ne connaît pas.

Pourtant, alors que l'on ne sait pas encore si l'autre est réellement humain - ou s'il pourrait jamais le devenir un jour - la contamination s'opère instantanément dès les premiers contacts, avant même que quiconque soit en état de comprendre l'autre. Au Brésil, les Français affamés par de longs mois en mer font signe du bateau aux «Sauvages » qui viennent à leur rencontre ; ils leur font comprendre qu'ils sont prêts à leur donner des objets en échange de nourriture, en leur montrant de loin «des couteaux, miroirs, peignes et autres baguenauderies ${ }^{7}$. On laisse immédiatement les Amérindiens monter à bord, parce qu'ils sortent de leurs pirogues les bras chargés de «farine faite d'une racine que les Sauvages mangent au lieu de pain, des jambons, et de la chair d'une certaine espèce de sanglier, avec d'autres victuailles et fruits à suffisance, tels que le pays en porte ${ }^{8}$. Les nôtres commencent par absorber la nourriture des "Sauvages"; bientôt ils boiront avec avidité leur caouin, fumeront voluptueusement leur petun ${ }^{9}$ et feront l'amour avec leurs femmes, qui ne font guère de difficulté à les accueillir. Puisqu'il est impossible de payer les «Sauvages" en argent, on leur donne «des chemises des couteaux, des hameçons de pêche, des miroirs et diverses marchandises et merceries ». Ils repartiront avec sur leurs canots, portant chemises et chapeaux de France.

\footnotetext{
${ }^{6}$ Jean de Léry, Histoire d'un voyage faict en la terre du Brésil (1578). 2ème édition, 1580. Texte établi, présenté et annoté par Frank Lestringant. Paris, 1994, Librairie générale française, p. 28.

7 Léry, op. cit., p. 148.

${ }^{8}$ Léry, op. cit., p. 148.

${ }^{9} \mathrm{Ou}$ kahui en langue tupi. C'est une boisson épaisse à base de racine de manioc mâchée et fermentée. Le voyageur allemand Hans Staden la trouve "épaisse, très enivrante et très nourrissante ». Le pétun est le nom du tabac en langue tupi. Léry, qui en a goûté, trouve que sa fumée « rassasie et garde d'avoir faim ».
} 


\section{L'échange inégal}

Dans ce marché par lequel des objets européens s'échangent contre des matières indigènes, l'autre qu'est le "Sauvage » ne connaît pas la valeur que représentent les biens qu'il obtient en échange de ceux qu'il offre. L'échange est donc biaisé et, évidemment, pour les Européens la tentation est grande d'abuser de l'autre, dont l'ignorance prend pour lui l'apparence de la stupidité. Dès les tous premiers contacts, en effet, les nouveaux arrivants découvrent que les "Sauvages» ne comprennent pas que ce qu'on leur propose en échange de leurs propres biens est en réalité sans grande valeur. Le "Sauvage », donc, donne tout ce qu'il a et prend tout ce qu'on lui offre, même si on lui présente des rebuts. Les Européens les mieux intentionnés prennent cela pour une générosité sans bornes; elle s'explique à leurs yeux par le fait que les "Sauvages » n'accordent de valeur à rien, pas même à leurs propres possessions. On peut donc tout leur prendre, pense-t-on, puisque cela n'a pas d'importance pour eux.

Or, si les Amérindiens donnent tout ce qu'on leur demande c'est parce que, ce faisant, ils font de celui qui reçoit d'eux leur allié et leur obligé : chez eux, à moins de se couvrir d'infamie, celui a reçu doit rendre le don par lequel il est lié à l'autre ; son honneur personnel et l'honneur du groupe tout entier auquel il appartient en dépendent. Pour l'Européen, la transaction s'arrête dès lors que la marchandise change de main. Pour l'Amérindien au contraire, elle ne fait que commencer. Jean de Léry a bien perçu que le don est chez les Tupinambas un mode d'échange ; il devine bien, en particulier, que celui-ci a pour fonction de cimenter la solidarité des individus à l'intérieur du groupe : "En se distribuant, et faisant journellement présent les uns aux autres, des venaisons, poissons, fruits et autres biens qu'ils ont en leur pays » écrit-il, les "Sauvages » ont systématiquement recours au don, car ils «mourrai(en)t de honte " s'ils voyaient leurs semblables manquer de ce qu'eux-mêmes possèdent, tout comme « les étrangers leurs alliés ».10

Dans ces pratiques de don, Léry ne voit pourtant que des actes de «charité » pratiqués à l'intérieur du cercle de la tribu et de ses alliés. Ce faisant, il tombe dans un travers bien connu de la pratique ethnographique, qui consiste à projeter inconsciemment sur l'étranger - en l'occurrence le «Sauvage » du Nouveau Monde - les codes de valeurs de sa propre culture; ainsi, les Topinambous seraient-ils naturellement " généreux » et " charitables », au sens commun où on l'entend dans l'Europe chrétienne du XVIe siècle. Au début du siècle suivant, le père capucin Yves d'Evreux, envoyé évangéliser les Tupinambas de l'île de Marahnam, à l'embouchure de l'Amazone, comprendra que, contrairement aux apparences, ces pratiques de don

${ }^{10}$ Léry, op. cit., p. 461. 
n'échappent nullement à la notion de valeur, dans la mesure où elles sont foncièrement des modes d'échange. C'est cependant d'un type de valeur bien particulier dont il est question ici : chez les Tupinambas, la valeur du bien cédé n'est autre que celle qu'elle a réellement pour son possesseur, et non, comme chez les Européens, celle que représente, en elle-même, son acquisition. Si l'un d'entre eux désire obtenir quelque chose qui appartient à l'un de ses semblables, écrit-il, «il lui dit franchement sa volonté, et il faut que la chose soit bien chère à celui qui la possède ». En retour, "si le demandeur a quelque chose que le donneur affectionne », celui-ci devra la lui céder "quand il la lui demandera $»^{11}$.

Plus de trois siècles plus tard, Claude Lévi-Strauss fera, chez les Tupi-Kawahib du Brésil, une expérience qui le replongera dans la situation décrite au XVIIe siècle par Yves d'Evreux. Alors qu'il lui est demandé de céder sa grande marmite d'aluminium dans laquelle il prépare tous les jours ses repas, et qu'il multiplie les explications justifiant l'impossibilité dans laquelle il se trouve de s'en séparer, il constate que plus il développe son argumentation et plus son interlocuteur Tupi affiche "un sourire épanoui, comme si mes paroles, dit Lévi-Strauss, répondaient à tous ses désirs ». Lorsque enfin l'ethnologue se trouve à bout d'arguments pour défendre sa possession, l'Amérindien s'empare sans façon de sa précieuse marmite et part avec, pleinement satisfait ${ }^{12}$.

Les hommes qui arrivent alors au Nouveau Monde n'entrevoient pas encore ces codes comportementaux, qui sont leur sont inaccessibles. En fait, Européens et Amérindiens se méprennent sur le statut qu'a l'échange chez l'autre et sur ce qui constitue la valeur des biens échangés. Pour les Européens, il est évident que l'on peut se servir à sa guise chez les "Sauvages » - en allant jusqu'à prendre les ressources qui les entourent, les objets qu'ils utilisent, et même les êtres avec lesquels ils vivent, puisqu'ils les offrent spontanément mais l'inverse, bien sûr, leur est totalement impensable. Car, si les Amérindiens possèdent collectivement des biens auxquels ils ne semblent attacher aucune valeur, les Européens sont persuadés de posséder individuellement des biens personnels, dont il serait parfaitement criminel de s'emparer. Il faut donc sévèrement punir ces indigènes qui, pensent-ils, abusent perfidement de leur confiance lorsqu'ils leur prennent quelque chose. C'est ainsi qu'à peine débarqués, les colons apportent avec eux l'injustice et la persécution, lesquelles sont engendrées par l'ignorance et l'incompréhension mêlées à la certitude qu'il n'existe qu'un seul droit et une seule vérité : les nôtres.

\footnotetext{
11 Yves d'Evreux, Suitte de l'Histoire des choses plus mémorables advenues en Maragnan es années 1613 et 1614. Second traité. Paris 1615, François Huby.

12 Lévi-Strauss, Tristes Tropiques, Paris, éditions Plon, 1955, p. 413-414.
} 
Les colons et les autochtones ne se comprennent pas mieux au sujet du travail. Travailler, au sens où l'entendent les Européens, est une notion parfaitement inconnue des «Sauvages ». Or, comme l'a largement montré Marshall Sahlins à propos des économies dites primitives, si les Amérindiens ne cherchent pas à rentabiliser leurs productions, $c^{\prime}$ est parce qu'ils ne le souhaitent pas et non pas parce qu'ils n'en sont pas capables ${ }^{13}$. Alors que les Européens s'activent à leurs affaires, les Amérindiens qu'ils rencontrent leur renvoient l'image de populations oisives, passant leur temps à danser et à « cahouiner »- c'est-à-dire à s'enivrer -, quand ils ne se font pas furieusement la guerre. Personne ne remarque qu' on n'y manque de rien. Surtout, personne ne voit le travail des femmes, qui, lorsqu'elles ne s'occupent pas des enfants et de la maison, cultivent la terre, portent les récoltes, ou encore fabriquent les pots et la plupart des objets domestiques.

Ainsi, aux yeux des Européens, les "Sauvages », que l'on a mis à l'ouvrage en les faisant exploiter le bois de brésil, ignorent toute notion de travail, dans la mesure où ils n'ont aucune idée de la valeur représentée par le labeur: pour les Européens qui les exploitent, les «Sauvages» leur apportent leur aide spontanément, en échange de biens de peu de valeur dont ils se satisfont. Au mieux, pense-t-on, ce sont des brutes innocentes et consentantes ; on peut donc les employer à des travaux de force, puisque cela ne les affecte pas. Au pire, ce sont des fainéants vicieux, qui doivent être éduqués et corrigés; il est donc juste de les forcer au travail, puisque cela ne représente rien pour eux. D'ailleurs, lorsqu'ils se plaignent, ce n'est pas de la peine qu'on leur impose, mais de ce que le système de travail forcé mis en place par les Européens les prive de prisonniers de guerre; c'est-à-dire d'une réserve suffisante de victimes pour perpétuer le cannibalisme rituel ${ }^{14}$. Pourquoi donc s'embarrasser de scrupules, considère-t-on, puisque, dans ces conditions, le travail ne peut représenter pour eux qu'un progrès bénéfique, qui les éloigne de leurs pratiques répugnantes?

\section{L'épuisement du Nouveau Monde}

Un système producteur d'inégalité, né de la collision de modes d'échanges inconciliables, prospère donc au Nouveau Monde. Le système de la traite, que $l^{\prime}$ on voit s'instaurer dès les premiers contacts, laisse une profonde impression d'injustice : alors que les Européens vont et viennent chargés de "cadeaux » que leur font continuellement les "Sauvages », ceux-là n'obtiennent que des

${ }^{13}$ Marshall Sahlins, Age de pierre, âge d'abondance. L'économie des sociétés primitives. Traduction française de Tina Jolas. Paris 1972, éditions Gallimard, collection Bibliothèque des Sciences humaines.

${ }^{14}$ Léry, op. cit., p. 353. 
objets de pacotille, quand ils ne sont pas complètement dépouillés par les Européens. "Beaucoup d'entre nous, rapporte ainsi Léry, allaient chargés d'objets personnels dont (les Tupinambas) nous avaient fait cadeau et qu'ils transportaient dans les filets où ils dorment: des plumets très riches, des arcs et des flèches en grand nombre ainsi qu'une infinité de perroquets de couleurs variées ».

Jusqu'à ce que les implantations européennes du Nouveau Monde soient suffisamment autonomes, ce sont les Amérindiens qui pourvoient d'autre part à l'alimentation des hommes débarqués des navires. Dans ce monde si différent du leur, les Européens sont incapables de produire de quoi pourvoir par eux-mêmes à leur existence. Ce sont les Amérindiens, surtout, qui, très rapidement, sont employés à leur service. Du Brésil, les Français rapportent essentiellement $\mathrm{du}$ bois de teinte, $\mathrm{du}$ coton et du poivre, ainsi que des perroquets et des petits singes, que leur procurent les Tupinambas 15 . Ils les obtiennent contre des tissus de couleur de bon marché, qui sont chargés en France lors du voyage aller des bateaux, et avec lesquels ils font confectionner des vêtements pour les indigènes ${ }^{16}$.

Pourtant, dès lors qu'ils sont transportés en Europe, ces matériaux ou ces animaux acquis à peu de frais en Amérique se convertissent en biens de luxe particulièrement recherchés. Ainsi, dès l'origine de la transaction avec les Amérindiens, l'échange est biaisé, car celui-ci repose sur la permutation de biens qui sont loin d'être équivalents. Fondamentalement, le «Sauvage » offre des ressources qu'il prend à son environnement et dont l'obtention lui coûte une somme de travail considérable: les hommes doivent abattre les aramboutan, ces arbres parfois gigantesques, qui donnent le bois de brésil ; ils doivent débiter les troncs et les transporter à dos d'homme jusqu'aux bateaux sur des distances atteignant parfois une quinzaine de kilomètres. Ils doivent en outre chasser intensivement les perroquets ou les singes; quant aux femmes, elles doivent passer de longues heures à cueillir le coton ou le poivre, ou encore à recueillir et à préparer les ingrédients de la nourriture offerte aux Européens... Contre ces ressources dont l'exploitation épuise leur environnement, les Tupinambas reçoivent des biens manufacturés dont les Européens n'ont pas directement besoin ou dont l'acquisition, en tout cas, ne leur a guère coûté d'efforts personnels : ils n'ont fait qu'acheter ces stocks de tissus, ces caisses d'outils, ou ces sacs de petites pièces de verroterie, qui leur

\footnotetext{
15 Hans Staden, Nus, féroces et anthropophages (Marburg, 1557). Traduction française d'Henri Ternaux-Compans. Paris 1979, éditions A.-M. Métaillé.

16 Comme l'indique Léry, "ayant porté dans nos navires grand quantité de frises rouges, vertes, jaunes et d'autres couleurs, nous leur en faisions faire des robes et des chausses bigarrées, lesquelles nous leur changions à des vivres, guenons, perroquets, brésil, coton, poivre long, et autres choses de leur pays, de quoi les mariniers chargent ordinairement leurs vaisseaux » (Léry, op. cit., p. 226).
} 
rapporteront un bénéfice sans commune mesure avec l'investissement consenti 17 .

On est frappé par le volume considérable des biens extraits du pays des Tupinambas: En 1531, la frégate La Pélerine ramène par exemple un chargement de quelques 3000 peaux de léopard, 300 singes et guenons et 600 perroquets «sachant déjà quelques mots de français ».18 En l'espace d'à peine quelques décennies, la présence européenne conduit irrémédiablement les Amérindiens à une surexploitation de leur environnement forestier, qui en épuise rapidement les ressources, foncièrement limitées. En raréfiant les ressources tout en augmentant la pression imposée au milieu, l'exploitation coloniale du Brésil ne fait pas que déstabiliser l'environnement; elle déstructure également les communautés indigènes. La surexploitation du milieu, en effet, amplifie les conflits entre des ethnies désormais en compétition pour l'obtention des ressources recherchées par les Européens. Ces guerres tribales sont exacerbées par la rivalité entre les Français et les Portugais, qui sont alliés à des tribus ennemies les unes des autres, et auxquelles ils fournissent des armes à feu dont le pouvoir de destruction est sans commune mesure avec celui de l'armement traditionnel des Amérindiens. Un mécanisme de désintégration à la fois environnementale et culturelle est donc enclenché par la présence européenne au Nouveau Monde.

$\mathrm{Au}$ moment où nous commençons à les connaître - vers le milieu du XVIe siècle - les populations amérindiennes du Brésil ne sont pas encore véritablement déculturées: elles entrent d'abord dans la dépendance économique des Européens - les Amérindiens ont désormais besoin des outils en métal fournis par les blancs pour survivre, d'un point de vue économique, dans la nouvelle économie de la traite - avant de tomber dans leur dépendance culturelle, prélude à leur disparition. L'épuisement de l'environnement, les guerres dévastatrices, les nouveaux enjeux de pouvoir, font bientôt exploser les cadres traditionnels de ces sociétés. Comme l'a montré en effet l'anthropologue Pierre Clastres, la structure de ces sociétés de chasseurs-cueilleurs d'Amérique du Sud vise à garantir la pérennité des groupes humains dans un environnement naturel aux ressources limitées. Elle s'oppose en particulier à l'accumulation de surplus économiques et au développement démographique des populations - qui introduisent l'un et l'autre le risque d'un épuisement irréversible du milieu - en maintenant, notamment par les guerres tribales, un morcellement maximum des

17 « Je vous laisse à penser à quelle peine (ce travail est réalisé par les Amérindiens) et ce pour appétit de gagner quelque pauvre accoutrement de méchante doublure ou quelque chemise ", écrit Thevet (op. cit., p 227).

18 Lévi-Strauss, op. cit., p. 91. 
communautés ${ }^{19}$. Or, c'est précisément ce que visent à empêcher ces sociétés qu'apporte l'installation des colons venus d'Europe. Et c'est la guerre qui, de régulatrice, devient destructrice dans le nouvel environnement créé par les Européens.

\section{Sois gentil, Français, donne-moi un bracelet de perles}

À l'approche du rivage, on tire un coup de canon du bateau, dont l'écho va se perdre dans l'immensité de la forêt. Les «Sauvages» sortent alors progressivement des bois, pour se rassembler sur la rive du fleuve. Les nôtres débarquent des coffres emplis de marchandises, les autres apportent de la nourriture. On échange. On discute, chacun apprenant le langage de l'autre.

- «Ere-ioubé ? Tu es venu?

- Pa-aiout. Oui, je suis venu.

- Teh! auge-ny-po. Voilà qui est bien dit. Ere-iacasso pienc? Tu as laissé ton pays pour venir rester ici ?

- Pa. Oui.

- Erérou dé caramémo ? Est-ce que tu as apporté tes coffres?

- Pà arout. Oui, je les ai apportés.

- Mobouy? Tu en as combien?

- Mocouein. Plusieurs.

- Maé pérérout, de caramémo poupé ? Et qu'est-ce que tu as apporté dans tes coffres?

- A-aub. Des vêtements.

- Mara vaé ? De quelles couleurs?

- Sobouy-eté. Il y en a des bleus. Pirenc. Des rouges. Ioup. Des jaunes. Tin. Des blancs. ${ }^{20}$

- Maé pamo ? Et quoi d'autre?

- A cang aubé-roupé. J'ai apporté des chapeaux.

- Seta-pé ? Tu en as beaucoup?

- Icatouvapé. Plus que tu ne pourrais en compter.

- Ai pogno ? Et c'est tout?

- Erimen. Mais non.

- Esse non bat. Alors montre-moi.

- Coromo. Attends un peu.

- Neîn. S'il te plaît; dis-moi.

- Bon : Mororocap. J'ai des arquebuses. Mocap-couiourou. Et aussi des cornes de poudre.

- Mâé pè sepouyt rem ? Qu'est-ce que tu voudras que je te donne pour les avoir?

- Arouri. Rien du tout ; je les ai juste apportées avec moi.

- Hé ! Allez!

- Karamoussee. Une autre fois.

- Tâcepiah taugé. Je voudrais les voir maintenant.

- Arrou-ita ygapen. J'ai aussi apporté des épées en fer...

- Nacepiac-icho péné ? Je peux les voir?

19 Pierre Clastres, La société contre l'État. Recherches d'anthropologie politique. Paris 1974, éditions de Minuit.

${ }^{20} C^{\prime}$ est-à-dire des chemises. 
- Bégoé irem. Un autre jour. Eémbereinguè. Attends un peu.

- Néréroùpe guya-pat? Et des serpes, tu en as apporté ?

- Arrout. Oui, j'en ai.

- Igatou-pé ? Elles sont belles?

- Guiapar-été. Elles sont excellentes.

- Ereroupè itaxé amo ? Tu n'as pas apporté de couteaux?

- Arroureta. J'en ai plein.

- Maé pamo ? Et quoi encore?

- Pinda. J'ai des hameçons. Montemonton. Des alènes. Arroua. Des miroirs. Kuap. Des peignes. Moùrobouy été. Des colliers ou des bracelets de verre bleu. Cepiah yponyéum. Des comme ça, tu n'en as encore jamais vus ; ce sont les plus beaux qu'on n'a encore jamais apportés ici.

- Easo ia-voh de caramemo t'acepiah dè maè. Ouvre ton coffre, que je voie ce qu'il y a dedans.

- Aimossaénen. Je ne peux pas.

- Nârour icho p'Irèmmaè desve ? Et toi, qu'est-ce que tu veux que je te donne?

- Mae! Pereou potat? Qu'est-ce que tu peux m'apporter?

- Sceh dè ; maé péréi potat? je ne sais pas ; qu'est-ce que tu veux?

- Pira, je voudrais du poisson. Ouy, de la farine. Commenda-ouassou, des grandes fèves. Commenda-miri, des petites fèves. Morgouia ouassou, des oranges et des citrons. Maé tirouèn, toutes sortes de choses différentes.

- Che-rorup-gatou, derour-ari. Je suis très heureux que tu sois venu. Nère roupé d'eré miceco ? Dis-moi, pourquoi tu n'as pas amené ta femme?

- Arrout iran-hèreco augernie. Je l'emmènerai quand j'en aurai fini avec mes affaires.

- Ty ierobah apoau ai. Tenons-nous glorieux du monde qui nous cherche. $»^{21}$

À terre, les femmes et les filles prennent le bras des étrangers venus de la mer et leur disent: "Mair, deogatorem, anabé mauroubi »; c'est-à-dire : "Sois gentil, Français, s'il te plaît donne-moi un bracelet de perles ». Des perles de verre bleu qu'on leur donnera, elles feront des parures qui remplaceront celles qu'elles fabriquaient traditionnellement en coquillage. Où qu'ils aillent, les Français sont suivis de bruyantes bandes d'enfants, qui leur répètent en riant : "Contoüassat, amabé pinda"; ce qui veut dire: "Mon ami et mon allié, donne-moi des hameçons pour la pêche ${ }^{22}$. Ils utiliseront ces accessoires en fer, qui remplaceront ceux qui étaient taillés jusqu'alors dans l'os ou la nacre. Ainsi, la technologie des Tupinambas se trouve-t-elle rapidement transformée par les outils en fer, que leur apportent les Français. Pour couper et trancher le bois de brésil, les Amérindiens se servent désormais de haches et de coins de fer, au lieu de l'outillage traditionnel de pierre. Mais, de plus en plus, ils emploient aussi des instruments en fer pour réaliser leurs productions traditionnelles d'ornements en plume. Ainsi, les Tupinambas du milieu du XVIe siècle fabriquent-ils encore les mêmes types d'objets, portant les mêmes genres d'ornementations traditionnelles que par le passé, mais ceux-ci sont

\footnotetext{
${ }^{21}$ Léry, op. cit., p. 479-489. L'ouvrage de Léry contient un premier dictionnaire des termes de la langue Tupinamba et un guide des expressions utilisées lors des échanges.

22 Léry, op. cit., p. 231, 233.
} 
désormais élaborés avec d'autres outils - ceux-là européens - qui en font des créations véritablement métisses.

En ce sens, l'introduction du fer dans ces populations de chasseurs-cueilleurs du Brésil dépasse le cadre d'un simple phénomène d'importation, dans la mesure où elle entraîne, dans la fabrication des objets de la culture matérielle traditionnelle, l'apparition de productions hybrides, mi-indigènes miétrangères. Les objets importés sont eux-mêmes adaptés, ou pourrait-on dire «traduits" dans leur milieu récepteur Tupi. Léry a noté ainsi que les Tupinambas prennent les couteaux à lame de fer qu'on leur offre, mais qu'ils en jettent les fourreaux, dont ils ne voient pas l'utilité. Il est très vraisemblable, par ailleurs, que les tissus de couleur apportés par les Européens - et notamment ces "chausses bigarrées » évoquées par Léry - aient été adoptés par les Amérindiens du Brésil en raison de leurs couleurs vives qui rappelleraient les rouges, les bleus et les jaunes éclatants de leurs parures de plumes traditionnelles.

En réalité, ces phénomènes d'adaptation et d'appropriation d'éléments de culture matérielle étrangère fonctionnent à double sens. Les Européens, qui tentent de recréer au Nouveau Monde l'univers matériel qu'ils ont laissé de l'autre côté de l'océan, se trouvent objectivement immergés dans la culture matérielle amérindienne. Dans la minuscule France antarctique, ils n'ont pour se nourrir que des aliments indigènes - comme de la farine de manioc, du poisson «boucané » et " $\mathrm{d}^{\prime}$ autres racines cuites aux cendres ${ }^{23}$ - nourriture à laquelle ils finissent par s'accoutumer ${ }^{24}$. Dans leur existence quotidienne, les colons utilisent aussi des accessoires amérindiens, qui remplacent désormais ceux qui leur étaient indispensables en Europe. Lorsque Jean de Léry et ses compagnons s'installent par exemple pour leur première nuit dans les baraquements venteux du fortin de Fort Coligny, ils n'ont pour couchette que des hamacs tupinambas : ce soir-là, écrit-il, «à la façon des Américains nous pendîmes des linceux (draps) et des lits de coton, pour nous coucher en l'air $\gg .{ }^{25}$

$\mathrm{Au}$ Nouveau Monde, les Européens qui débarquent de leurs vaisseaux sont avides de tout: d'abord de nourriture, de femmes, mais surtout $\mathrm{d}^{\prime}$ informations. Les gens d'ici ont-ils vu d'autres hommes blancs avant eux ? Connaissent-ils d'autres fleuves, d'autres contrées, d'autres peuples ? Est-ce loin d'ici, par où y va-t-on ? Savent-ils si l'on trouve de l'or par ici ? Qui en possède parmi eux? Les tous premiers mots qui s'échangent d'une langue à

\footnotetext{
${ }^{23}$ Léry, op. cit., p. 164.
}

${ }^{24}$ Léry rapporte ainsi qu'à leur retour en France, certains ses compagnons avaient "pris le vin en tel dégoût, qu'ils furent plus d'un mois sans en pouvoir sentir, encore moins goûter. » (Léry, op. cit., p. 547)

${ }^{25}$ Léry, op. cit., p. 164. 
l'autre sont ceux qui servent à poser ces questions : comment s'appelle cet objet, cet outil ? Quel nom a cet aliment? Les nouveaux mots qui surgissent sont ceux qui donnent le nom des choses que l'on a besoin de savoir pour obtenir de l'autre ce que l'on est venu chercher si loin. A force d'être répétés, ces mots s'incrustent dans le langage et s'y adaptent. Sous l'impact des termes venus de l'autre bout du monde, le vocabulaire se créolise. Certains de ces anciens mots américains se sont installés dans notre langue : les Tupinambas du Brésil nous ont donné topinambours, manioc, goyave, cajou, ananas... Mais qui se souvient encore que le terme caraïbe, donné aux premières populations amérindiennes rencontrées par Colomb, vient de ce qu'elles avaient coutume d'accueillir les étrangers par le mot "Caraiubé », lequel signifiait, dans leur langue, «bonne vie » ou « soyez le bienvenu » ?26

\section{« Des bêtes à figure humaine »}

Les images somptueuses des Grands voyages de Théodore de Bry nous montrent les «Sauvages d'Amérique» sous l'apparence de colosses nus évoquant les figures des dieux de l'Antiquité, mais armés d'arcs et de massues en bois. Ces guerriers sauvages arrivent au-devant des bateaux européens sur des multitudes de pirogues, portant des parures de plumes aux couleurs éclatantes : ce sont $d$ 'insolites couronnes ou coiffes de plumes verticales, et des ornements de plumes rayonnantes retenus par un lien passé en bandoulière, que les guerriers portent sur le côté des reins ${ }^{27}$. Dans les danses cérémonielles qu'on les voit accomplir ensemble, ils sont parés d'étranges capes de plumes et de jarretières de plumes passées au mollet. Leur corps est couvert d'un fin duvet de plume teint en rouge par du brésil, qui dessine sur leur peau des taches de couleurs vives. Le devant de leur crâne est rasé, de même qu'à l'arrière de la tête le haut de la nuque. Leur corps, souvent peint en noir, est complètement épilé.

La lèvre inférieure des hommes est percée: les adolescents, indique Léry, portent un petit ornement d'os poli, en forme de quille. Il est remplacé, lorsque ceux-ci deviennent adultes, par une grosse pierre verte « de la rondeur et largeur et deux fois plus épaisse qu'un teston $28 »$. Autour du cou, les hommes portent « des croissants plus longs que demi-pied, faits d'os bien unis, aussi blancs qu'albâtre, lequel ils nomment Y-aci du nom de la lune, qu'ils appellent

\footnotetext{
26 Thevet, op. cit., p. 113.

${ }^{27}$ Ces «pennaches » sont appelés Araroye en langue tupi. Léry, op. cit., p 223. Théodore de Bry, Le Théâtre du Nouveau-Monde. Les Grands Voyages de Théodore de Bry. Présenté par Marc Bouyer et Jean-Paul Duviols. Paris 1992, éditions Gallimard, collection Découvertes Gallimard Albums.
}

${ }^{28}$ Léry, op. cit., p. 216. 
ainsi ${ }^{29}$, ainsi que des colliers de disques en coquillage poli, appelés Boüre. La parure des femmes semble toujours plus discrète, en regard de celle, flamboyante, des guerriers. Elles ne portent éventuellement qu'un collier de petits coquillages. Leurs longs cheveux noirs sont enroulés en nattes et maintenus par des fils de coton teint en rouge. Sur les épaules, elles jettent parfois de minces filets dans lesquels elles portent les enfants dans leur dos (fig. 2).

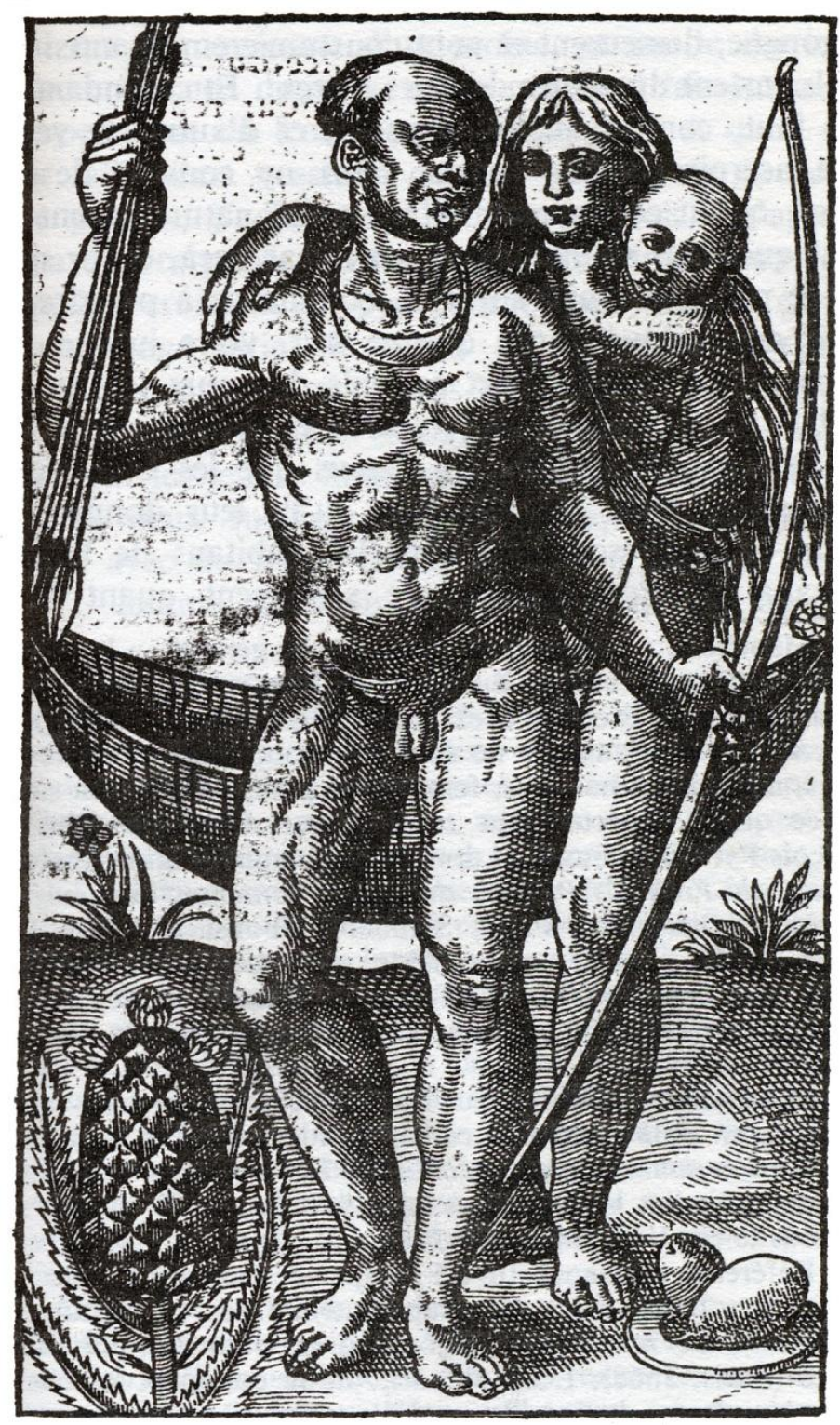

Fig. 2. Famille d'Indiens du Brésil. XVIè siècle. LERY, Jean de. Histoire d'un voyage fait en la terre du Brésil, Autrement dite Amérique, 1578.

Partout où ils les rencontrent, les Européens sont frappés d'abord par la nudité des "Sauvages" du Nouveau Monde qui s'expose à eux sans retenue. Le contact avec le corps nu des Amérindiens est peut-être le plus étroit au Brésil,

${ }^{29}$ Léry, op. cit., p. 219. 
où tout Européen s'égarerait presque immédiatement dans la forêt s'il perdait un instant du regard le puissant dos nu des hommes indigènes qui les précèdent en guidant leurs pas, ou même qui les portent sur leurs épaules, comme des petits enfants. Bien que quatre siècles les séparent, les gravures des Grands voyages de Théodore de Bry et les photographies en noir et blanc rassemblées par Lévi-Strauss dans ses Tristes Tropiques portent la marque de la même fascination pour la nudité amérindienne. Sur ces images, on voit les hommes de dos, assemblés pour des danses, solidement plantés sur leurs jambes et portant leurs parures étranges et belles de longues plumes aux couleurs éclatantes. Les femmes sont complètement nues. On les observe qui bavardent entre elles négligemment allongées dans des hamacs, ou qui dorment couchées directement dans la poussière, comme ne le font chez nous que les bêtes, les clochards et les ivrognes. Depuis le XVIe siècle, les Européens emmitouflés dans leurs vêtements sont travaillés par la tentation de détailler le corps des femmes amérindiennes abandonnés à leurs regards. Comme LéviStrauss au travers de son appareil photographique, ils les contemplent à la dérobée lorsqu'elles dorment, couchées sur des nattes ou étendues directement sur la terre rouge au milieu des débris d'écorce, parfois avec un petit enfant nu enlacé à elles, sa petite main délicatement posée sur un de leurs seins (figure 3). Il émane du corps de ces femmes et de ces filles sauvages une expression de liberté impensable, pour ne pas dire insupportable. C'est une liberté dont on ne sait si elle est admirable ou au contraire haïssable.

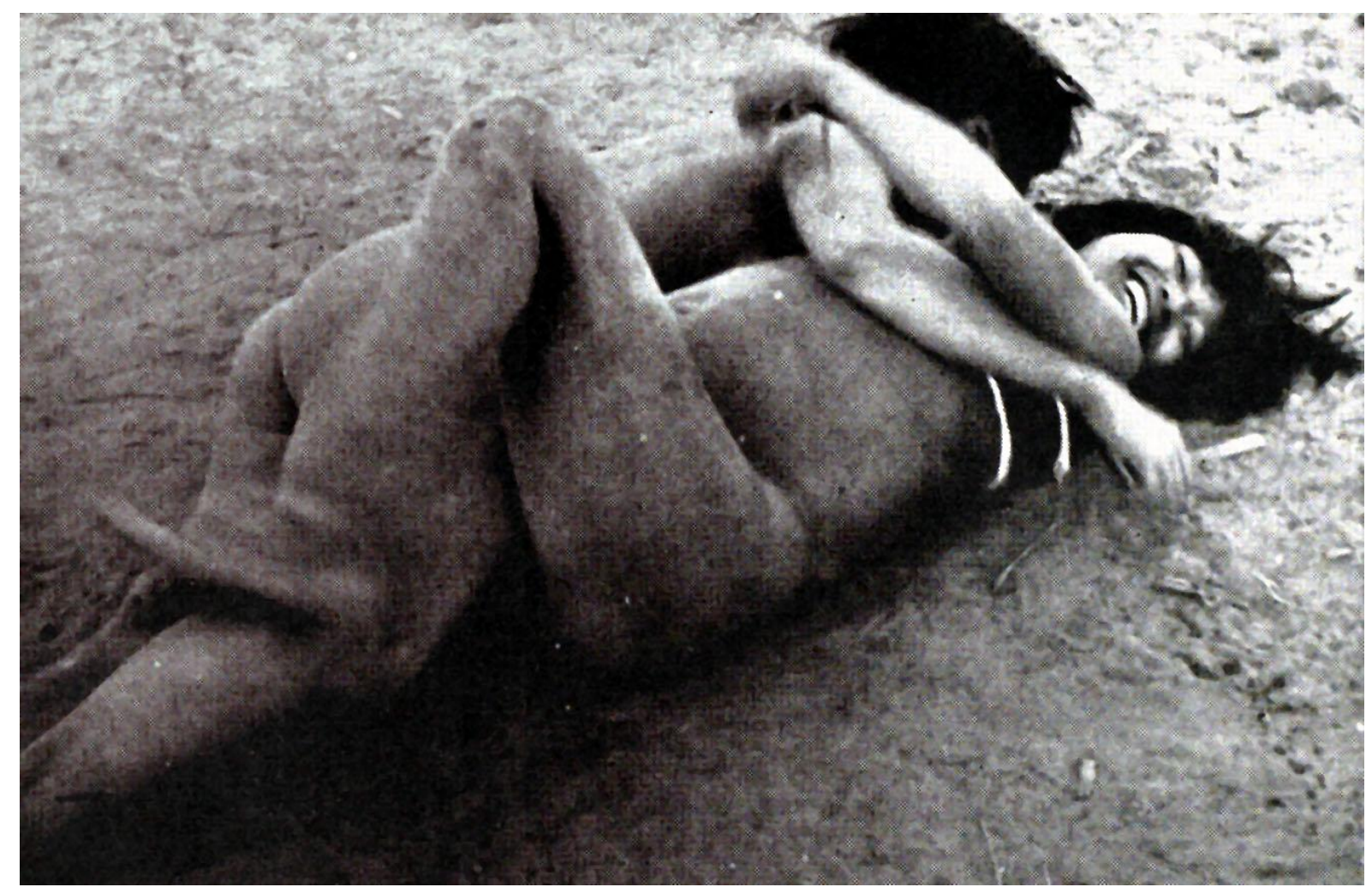

Fig. 3. « À deux » - Claude Lévi-Strauss,. Saudades do Brasil, 1994. 
Car, au détour d'une case, la beauté peut en un instant se tourner en horreur : entre eux, les «Sauvages » se repaissent de chair humaine. Les illustrations de Théodore de Bry, qui illustrent les épisodes du séjour forcé d'Hans Staden, prisonnier pendant de longs mois des Amérindiens du Brésil, font de l'anthropophagie le cœur de l'activité sociale des «Sauvages». Toutes les étapes du processus de la dévoration de l'homme y sont représentées en détail, depuis la mise à mort des victimes, la préparation du corps, puis sa découpe, la cuisson des morceaux, et enfin leur consommation collective. Ce sont les femmes, belles et nues, qui sont montrées préparer les corps des victimes - évidemment masculins - et porter les morceaux à cuire. Les guerriers tuent et coupent. Sur une des planches les plus étonnantes de Théodore de Bry, une assemblée de «femmes sauvages » est réunie : elles sont représentées accroupies autour de plats (?) sur lesquels est disposée, bien en évidence, une tête d'homme coupée et des entrailles, dont l'une d'entre elles, qui porte un petit enfant sur le dos, se saisit avec avidité. Cette assemblée d'ogresses se délecte d'abats difficilement identifiables, qu' elles partagent avec leurs petits. Femmes et enfants portent à leurs lèvres des coupes qui contiennent, selon de Bry, "une espèce de bouillon (de viande humaine) nommé mingau ».

Ces gravures troublantes, réalisées à partir des indications de Staden, révèlent au public européen l'indicible sauvagerie des hommes du Nouveau Monde. Elles visent à en restituer, au plus près, la vérité. Il ne s'agit plus, ici, d'histoires de monstres fabuleux mais d'une situation authentique, attestée par des témoins dignes de foi qui l'ont vue de leurs propres yeux. Comme le rapporte en effet Staden :

Les femmes prennent les entrailles, les font cuire, et en préparent une espèce de bouillon nommé mingau, qu'elles partagent avec leurs enfants; elles dévorent aussi les entrailles, la chair de la tête, la cervelle et la langue ; les enfants mangent le reste. Aussitôt que tout est terminé, chacun prend son morceau pour retourner chez lui. J'ai vu toutes ces cérémonies et $j^{\prime} y$ ai assisté. 30

Le paradis du Nouveau Monde se révèle en réalité un enfer. Une planche des Grands voyages de Théodore de Bry représente le voyage de Léry au Brésil. L'œil domine la scène, au premier plan de laquelle Léry et un de ses compagnons tentent de persuader un «Sauvage» nu de renoncer à ses horribles pratiques païennes : l'index levé au ciel, le protestant lui indique la place où se tient Dieu, auquel tous les hommes sur terre doivent foi et obéissance. L'homme qui l'accompagne nous tourne le dos. La main portée à son visage, il considère avec effroi le spectacle de désolation qui s'étend derrière les deux hommes, sur la terre brésilienne. Ce monde est maudit : des démons grimaçants, griffus et cornus, assaillent les «Sauvages » pendant leur

${ }^{30}$ Le témoignage de Staden est publié en 1557 à Marbourg (Hans Staden, op. cit.). 
sommeil et les poursuivent pour les flageller. Dans le ciel volent lourdement des monstres à corps de serpent ou à ailes de vampires. La mer toute proche, où mouille un vaisseau européen, est infestée de poissons énormes et effrayants. À l'arrière-plan, se serrent les pauvres cabanes de bois et de feuilles des Tupinambas, qui sont plongées dans cet univers de cauchemar. Au contraire des Européens, qui sont protégés par leur foi en Dieu, les "Sauvages» sont livrés aux démons par leur bestialité impudique. Le Nouveau Monde est un pays sauvage, donc diabolique. Et les hommes qui peuplent cette terre de perdition ne sont pas des hommes, mais des êtres qui n'ont d'humain qu'une ressemblance physique superficielle avec la véritable humanité, qui est la nôtre. Villegagnon, le créateur despotique de la colonie huguenote de Fort Coligny, avait déjà dit des Amérindiens brésiliens qu'il y avait rencontrés : (ce sont) « des gens farouches et sauvages, éloignés de toute courtoisie et humanité, du tout différents de nous en façon de faire et instruction : sans religion, ni aucune connaissance d'honnêteté ni de vertu, de ce qui est droit ou injuste ». Et il ajoutait : « en sorte qu'il me venait en pensée, à savoir si nous étions tombés entre des bêtes portant la figure humaine » 31

\section{Repousser l'ensauvagement}

Des monstres bestiaux affublés d'une apparence humaine : tels sont, pour les Européens, les «Sauvages » du Nouveau Monde. À leurs yeux, il est essentiel d'éviter de se lier, autant que faire se peut, avec ces êtres dépourvus de toute idée de morale. Cela est d'autant plus nécessaire qu'en réalité la « sauvagerie » tend insidieusement à gagner tout le monde. On commence par adopter les modes de vie des «Sauvages" - à s'acclimater à leur nourriture, à dormir comme eux dans des hamacs... - puis, insensiblement, on en est conduit à se compromettre avec eux. Léry dénonce ainsi le comportement des Français qui se lient avec des «putains » indigènes, manifestement nombreuses aux abords du petit établissement français ${ }^{32}$. Le maintien d'une absence de mixité avec les «Sauvages » est considérée comme vital pour la conservation de l'intégrité de la communauté d'origine française, à telle enseigne qu'à Fort Coligny, Villegagnon punit de mort tout chrétien qui se mettrait en ménage avec une femme indigène.

Pourtant, certains, parmi les Français expatriés, en viennent à préférer la vie libre que mènent les «Sauvages » dans la forêt à l'existence misérable qu'ils

\footnotetext{
${ }^{31}$ Léry, op. cit., p. 69. Thevet lui-même dit des Amérindiens du Brésil qu'il s'agit de "gens merveilleusement étranges et sauvages, sans foi sans loi, sans religion, sans civilité aucune, mais vivant comme bêtes irraisonnables, ainsi que la nature les a produits, mangeant racines, demeurant toujours nus, tant hommes que femmes... (Thevet, op. cit., p. 122).

${ }^{32}$ Léry, op. cit., p. 180 et 71.
} 
sont condamnés à mener dans le simulacre de civilisation de Fort Coligny. Ceux-là ne tardent pas à abandonner la communauté divisée des colons, pour les rejoindre. Léry rapporte ainsi qu'un groupe d'hommes de la petite colonie française, ne supportant plus les mauvais traitements que leur faisait subir Villegagnon, s'est enfui dans la forêt pour retrouver les "Sauvages». Ils " aimèrent mieux, écrit Léry à propos de ces fugitifs "plus maltraités qu'aux galères ", s'aller rendre en terre ferme avec les sauvages (lesquels aussi les traitaient plus humainement) que de demeurer davantage avec lui »33. Ailleurs, c'est un groupe de marins normands, survivants d'un naufrage, qui s'est établi depuis plusieurs années chez les Tupinambas, où ils ont pris femme et fondé des familles. Là, dit Léry, "vivant sans crainte de Dieu, ils paillardaient avec les femmes et filles (comme j'en ai vu qui en avaient des enfants déjà âgés de quatre à cinq ans). »34

Des groupes d'Européens sont donc délibérément établis chez les "Sauvages", d'où ils ont décidé de ne plus jamais revenir. Parmi eux se trouvent également des "truchements»; c'est-à-dire des jeunes gens, qui, orphelins ou abandonnés, ont été envoyés lorsqu'ils étaient encore enfants dans les communautés Tupinambas afin d'en apprendre le langage - en quelque sorte comme une seconde langue maternelle - et servir ainsi $\mathrm{d}$ 'interprètes auprès des Français. Lorsque Léry se rend au Brésil, les " truchements » qu'il y rencontre sont établis, pour certains, depuis déjà neuf ou dix ans. ${ }^{35}$ Devenus adultes, beaucoup d'entre d'eux ont désormais vécu plus longtemps dans la forêt brésilienne qu'au contact de la civilisation, laquelle n'est plus guère pour eux qu'un souvenir incertain de la petite enfance. Ils se sont «ensauvagés » et sont devenus, pour la plupart d'entre eux, des Tupinambas à la peau blanche. Léry cite ainsi le cas de plusieurs "truchements» originaires de Normandie, installés chez les Tupinambas depuis huit ou neuf ans, lesquels, dit-il,

menant une vie d'Athéiste, ne se polluaient pas seulement en toutes sortes de paillardises et vilenies parmi les femmes et les filles, dont un entre autres de mon temps avait un garçon âgé d'environ trois ans, mais aussi, surpassant les sauvages en inhumanité, j'en ai entendu qui se vantaient d'avoir tué et mangé des prisonniers. ${ }^{36}$

Dans ce discours de dénonciation de l'ensauvagement, ce sont les femmes indigènes qui sont clairement désignées comme les responsables de la perte des Européens : elles ne sont que des "putains », qui les attirent sexuellement et leur font bientôt des enfants, afin de mieux les entraîner dans une vie de débauche qui les amènera à renier leur foi. Car ce sont les femmes indigènes,

\footnotetext{
${ }^{33}$ Léry, op. cit., p. 189.

${ }^{34}$ Léry, op. cit., p. 180.

${ }^{35}$ Léry, op. cit., p. 79.

${ }^{36}$ Léry, op. cit., p. 370.
} 
rappelle Léry, qui rejettent les vêtements qu'on leur donne pour cacher leur nudité et qui s'exhibent toutes nues la nuit, où elles retournent à leur sauvagerie impudique ${ }^{37}$. Aux yeux de tout bon chrétien, les femmes indigènes sont mauvaises, parce qu'elles sont irréductiblement sauvages et parce qu'elles menacent, par leur simple contact, l'intégrité morale des hommes civilisés.

Ainsi, un racisme dirigé contre les «Sauvages » prospère-t-il à l'intérieur de la petite colonie d'expatriés établis en France antarctique. Ce racisme «antisauvages ", exprimé notamment par les Français, se développe dès lors qu'un rapport de domination sur les Amérindiens se trouve installé, en particulier à partir du moment où s'instaure un système d'exploitation des ressources de la forêt brésilienne effectué par les Amérindiens au profit des Européens. On considère désormais leurs particularités culturelles (comme aller nu, échanger des biens dans le cadre d'une économie du don, pratiquer l'anthropophagie, ne pas croire en Dieu, etc...) comme des pratiques déviantes, qui doivent être incessamment réprimées et qui, de toutes façons, les identifient comme des êtres non civilisés : dans cette configuration, ou bien les "Sauvages" du Nouveau Monde sont assimilables, et dans ce cas ils sont destinés à devenir des Chrétiens de seconde zone; ou bien ces êtres déviants ne sont pas assimilables et dans ce cas ils ne sont qu'une forme d'humanité inférieure : une espèce qui - pour le bien de la véritable humanité ; à savoir la nôtre - nécessite impérativement d'être annihilée.

\section{Comment peut-on être Sauvage?}

Mais qui donc sont vraiment ces «Sauvages américains »? Peut-on connaître ces gens, au-delà de leur immédiate apparence d'étrangeté, et, dans ce cas, qu'en dire? Rien n'est moins sûr, et les observateurs qui les côtoient suffisamment longtemps pour en devenir familiers avouent leur impuissance tel, le premier, Léry: " À cause de leurs gestes et contenances du tout dissemblables des nôtres, je confesse, écrit-il, qu'il est malaisé de les bien représenter, ni par écrit ni même par peinture. $38_{\text {» }}$ Rien de ce que nous connaissons dans nos sociétés occidentales ne trouve apparemment de correspondance chez eux, en sorte qu'il n'est guère possible de les identifier que par la liste de tout ce qui leur fait défaut, ainsi que l'écrira Michel de Montaigne :

C'est une nation, dirais-je à Platon, en laquelle il n'y a aucune espèce de trafic [commerce]; nulle connaissance des lettres; nulle science des nombres; nul nom de magistrat, ni de supériorité politique ; nul usage de service [servitude], de richesse ou

${ }^{37}$ Léry, op. cit., p. 231-232.

${ }^{38}$ Léry, op. cit., p. 234.

Heródoto, Unifesp, Guarulhos, v. 3, n. 1, Março, 2018. p. 563-587 
de pauvreté ; nuls contrats ; nulles successions ; nuls partages ; nulles occupations, qu'oisives ; nul respect de parenté, que commun ; nuls vêtements ; nulle agriculture ; nul métal ; nul usage de vin ou de blé. Les paroles mêmes qui signifient le mensonge, la trahison, la dissimulation, l'avarice, l'envie, la détraction [médisance], le pardon: inouies. ${ }^{39}$

Les Amérindiens du Brésil représenteraient donc la somme de tout ce que nous ne sommes pas et plus exactement la négation de ce que nous considérons être la culture et la civilisation. Dans ces conditions, comment les comprendre, autrement que comme des êtres non civilisés, c'est-à-dire, à proprement parler, des sauvages ? Et surtout, comment les aborder, autrement qu'à travers le filtre de nos propres conventions culturelles ?

Les Amérindiens, ainsi, fascinent. Tout au long du XVIe siècle, on a ramené en Europe de nombreux groupes de "Sauvages d'Amérique ». Dès 1509, sept Amérindiens béothuks du Canada débarquent en France avec le navigateur Thomas Aubert, parti l'année précédente de Dieppe pour un voyage de pêche et d'exploration ${ }^{40}$. Ce sont sans doute les premiers «Sauvages » du Nouveau Monde jamais vus en France ${ }^{41}$. Parés de plumes et portant leur canoë d'écorce sur le dos, ils défilent dans les rues de Rouen, où ils créent l'étonnement. L'exhibition des "Sauvages » devient rapidement un spectacle. En 1550, la ville de Rouen accueille une extraordinaire fête brésilienne organisée pour la visite du roi Henri II et son épouse Catherine de Médicis. À l'extérieur des fortifications, la forêt toujours verte du Brésil a été reconstituée, avec des arbres étoffés de rameaux de buis auxquels on a accroché des répliques des fruits magnifiques de la France antarctique. On y a placé aussi des animaux de l'Amérique, comme des singes et des perroquets. Une troupe de plus de trois cent figurants nus - parmi lesquels figure une cinquantaine de véritables Indiens Tupinambas - y donne une représentation des scènes de la vie sauvage de la «France antarctique». Les spectateurs les voient chasser les animaux, commercer le bois de brésil avec les marins français et surtout repousser victorieusement une attaque de leurs terribles ennemis Tabajaras, alliés des Portugais. Devant le succès remporté par le spectacle, deux autres

\footnotetext{
${ }^{39}$ Montaigne, Les Essais, Livre Ier, chapitre 31 (des cannibales), Paris 2002, éditions Arléa, p. 159.

${ }^{40}$ Le peuple des Béothuks s'est éteint au début du XIXe siècle, avec le décès de Shanawdithit, la dernière représentante connue de ce groupe autochtone de Terre Neuve.

${ }^{41}$ Vicente Pinzon, qui longea les côtes du Brésil en 1499, avait ramené au Portugal un groupe de vingt Amérindiens, ayant survécu à la traversée. Peu après lui, Diego de Lepe ramena à Séville des esclaves amérindiens, également originaires du Brésil. En 1501, Vespucci avait ramené trois Amérindiens du Brésil au Portugal pour qu'ils apprennent la langue et puissent servir d'interprètes aux Portugais.
} 
fêtes avec des "Sauvages d'Amérique » seront organisées en 1564, pour la visite du roi Charles IX à Troyes, puis en 1566 à Bordeaux ${ }^{42}$.

Comme les singes et les perroquets rapportés du Brésil, on distribue des petits «Sauvages » en cadeau. En 1558, lors du voyage de retour de Léry en France, un groupe de dix jeunes garçons est envoyé au roi Henri II. 43 Ce sont des esclaves, qui ont été sélectionnés parmi la cinquantaine de prisonniers de guerre achetés par Villegagnon aux Tupinambas pour travailler à Fort Coligny. Le monarque les distribue comme présent royal à ses seigneurs de la cour. Quelques années plus tard, en 1562, Montaigne rencontrera à Rouen trois Amérindiens Tupinambas débarqués du Brésil, qui sont présentés au jeune roi Charles IX, lequel n'a encore qu'une douzaine d'années. Montaigne tirera de cette entrevue extraordinaire les réflexions développées dans son célèbre Essai sur les cannibales. ${ }^{44}$

Nul ne sait combien d'Amérindiens du Brésil ont été transportés en Europe comme curiosités exotiques, comme esclaves ou comme visiteurs qui ne sont jamais repartis. Débarqués en France, certains indigènes du Nouveau Monde y fondent des familles franco-amérindiennes. L'un des tous premiers dont on connaisse quelque peu l'histoire s'appelait Essomeriq. Ce jeune fils d'un chef amérindien des côtes du Brésil fut embarqué en juillet 1504 par le capitaine Binot Paulmier de Gonneville à bord de son vaisseau l'Espoir, qui retournait vers Honfleur, son port d'attache. Ayant failli périr durant la traversée, Essomeriq fut baptisé par le capitaine, qui lui donna son nom, Binot. Revenu en Normandie, le capitaine Gonneville l'éleva comme son propre fils. Lorsque Binot eut atteint l'âge de trente ans, son père adoptif le maria à sa fille Suzanne. À sa mort, le généreux capitaine lui abandonna tous ses biens par testament à condition que Binot porte toujours le nom de Gonneville et transmette les armoiries de sa famille ${ }^{45}$. Quelques années plus tard, en 1529, six Amérindiens, qui avaient été abandonnés par les Portugais sur l'île de Sainte-Hélène, furent embarqués par un vaisseau français, qui les déposa à Dieppe. On perd rapidement la trace de la plupart d'entre eux. Cependant, l'un de ces Amérindiens Tupi, s'établit à Dieppe, et s'y maria. On peut supposer qu'il y trouva une activité et qu'il y acquit des biens. Il mourut à Dieppe en 1569, après quarante années passées dans la ville ${ }^{46}$.

\footnotetext{
42 Ferdinand Denis, Une fête brésilienne célébrée à Rouen en 1550. Paris 1850, J. Techener.

${ }^{43}$ Léry, op. cit., p. 353.

44 Montaigne, op. cit., Livre Ier, chapitre 31 (des cannibales), p. 156-164.

45 Binot Paulmier de Gonneville, Le Voyage de Gonneville (1503-1505) et la découverte de la Normandie par les Indiens du Brésil. Paris 1995, éditions Chandeigne. L'aventure d'Essomeriq est relatée également par Paul Gaffarel, Histoire du Brésil français au XVIe siècle. Paris 1878, Maisonneuve, $\mathrm{p} 30-54$.

${ }^{46}$ Afonso Arinos de Melo Franco, op. cit., p. 84.
} 


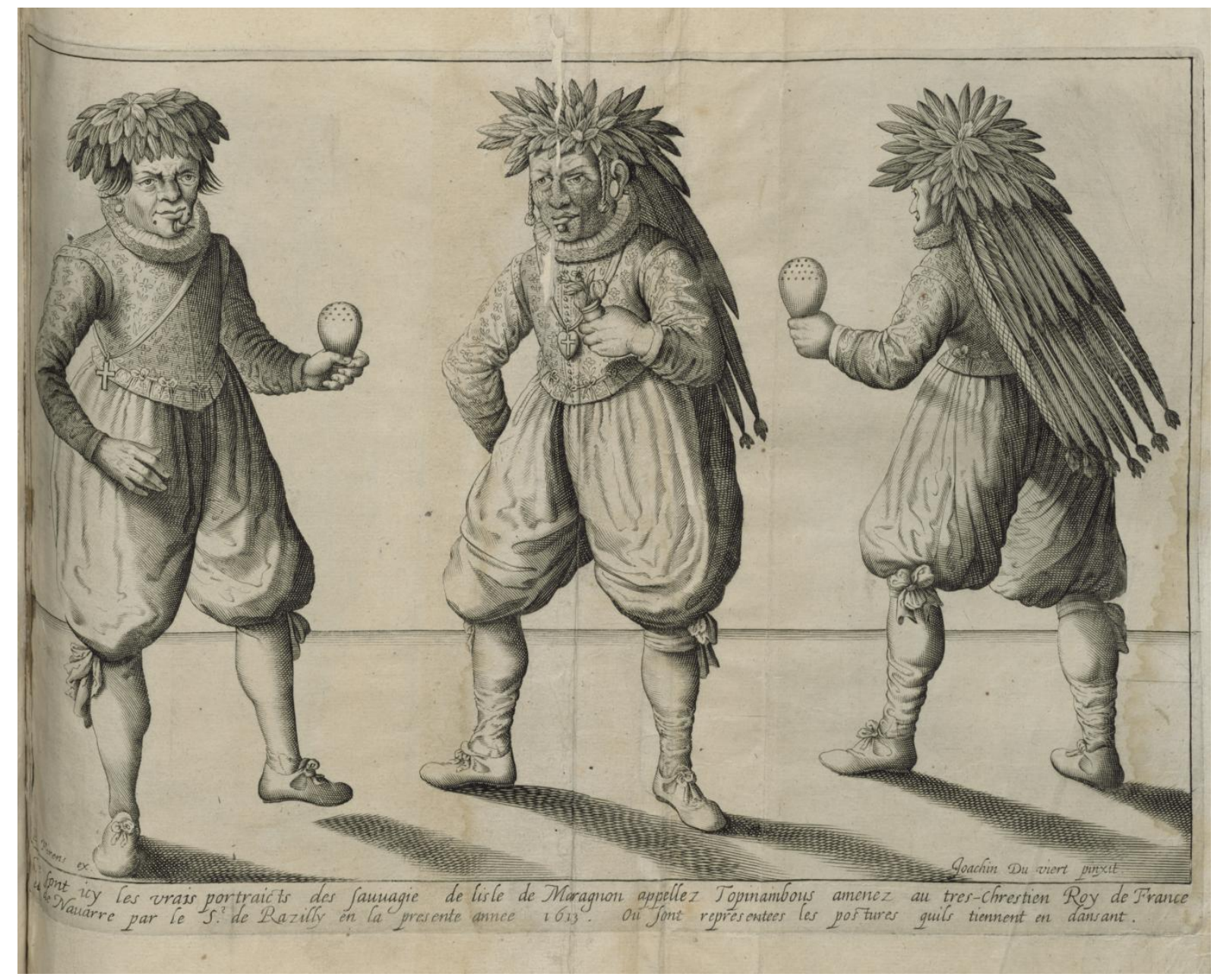

Fig. 4. Les Tupinambá en France, Joachim du Viert et Pierre-Jean Mariette, 1613.

Dans tous les cas, on ne sait pas ce que ces Amérindiens établis en France devinrent par la suite. Leur arrivée, à la descente du bateau, est remarquée ; ils suscitent la curiosité de la foule tant qu'ils ont l'air encore à demi-sauvages et qu'il reste une marge suffisante pour les assimiler - en les habillant comme nous, en les baptisant ou en les mariant comme nous-mêmes (figure 4). Mais lorsque tout cela est finalement accompli, leur sort n'intéresse plus personne. Ils ne sont plus que des étrangers parmi d'autres, et ne font plus jamais parler d'eux. Leurs enfants métis, déjà, ne possèdent plus rien du monde dont euxmêmes ont été extraits. Pour qu'ils s'intègrent enfin parmi nous, il est nécessaire qu'ils oublient qui ils sont et d'où ils viennent.

\section{« Notre monde vient d'en trouver un autre... »}

De ce passé maintenant lointain, il nous reste ces histoires, qui nous touchent encore aujourd'hui car elles disent un futur impossible, qui se noue dans l'instant suspendu d'une rencontre, dont on comprend bien qu'elle ne peut 
pas durer. Les univers des «Sauvages» et des «Civilisés » sont inconciliables et cette confrontation, dont on peut croire encore à l'innocence de cet instant, signe la fin d'un monde : les étrangers qui débarquent apportent avec eux la dépossession et la domination, qui ne tarderont pas à s'installer. On se dit qu'une conjonction miraculeuse s'est produite à ce moment, aujourd'hui si loin de nous, et qu'en même temps cela ne pouvait pas advenir, parce que nous étions, sans l'être encore, déjà ce que nous sommes devenus. Nous avons été bouleversés par cette rencontre avec les «Sauvages » du Brésil, qui n'aura duré que l'espace de quelques années, mais dont nous conservons encore l'étonnement, comme Léry revenu parmi les siens en France, «une blessure étrange dans le cœur ».

Car l'Autre est insaisissable. Comme les vestiges ténus du passé qui se désagrègent quand on les met au jour, il n'est pas possible d'aborder les «Sauvages », de les toucher, sans les défaire du même coup. À notre contact, ils se dissolvent et "s'évanouissent comme un songe », ainsi que l'avait déjà noté Plutarque à propos des Gaulois, vaincus et colonisés par les Romains. ${ }^{47}$ Ce monde disparu a néanmoins la persistance fugitive des images des rêves. Nous l'avons entrevu, dans un autrefois révolu et désormais étranger, dont la présence invisible continue à travailler secrètement notre présent. Nous l'avons perdu, mais il en reste des impressions, ensevelies dans les profondeurs de la mémoire collective.

« Notre monde vient d'en trouver un autre »; c'est ainsi que, dans les années 1580, Montaigne prenait note, dans ses Essais, de la découverte du Nouveau Monde pour constater qu'à peine l'avions-nous rencontré, nous avions déjà mutilé et trahi ce «monde enfant» qui s'offrait à nous ${ }^{48}$. La prise de possession des Amériques constitue un extraordinaire moment de confrontation avec l'Autre, tel que l'Occident n'en avait pas connu depuis l'Antiquité. Car ce ne sont pas de simples étrangers que rencontrent les voyageurs de l'autre côté de l'océan: ce sont des êtres ignorant tout ce qui constitue, à nos yeux, les fondements mêmes de l'humanité et en même temps des gens identiques à nous-mêmes, capables d'éprouver absolument les mêmes sentiments que les nôtres ${ }^{49}$. Aussi, cette découverte des "Sauvages américains » non seulement bouleverse l'idée que nous nous faisions de l'Autre, mais elle modifie également profondément le regard que nous portions sur nous-mêmes comme elle ruine la fiction de nos origines fondées

\footnotetext{
47 Plutarque, Vie de César, 27, 1-8.

48 Michel de Montaigne, Les Essais, Livre III, chapitre VI (des coches). Paris 2002, éditions Arléa, p. 658-662.

${ }^{49}$ Ton Lemaire, De Indiaan in on bewustsijn. De ontmeting van de Oude net de Nieuwe Wereld (The Indian in our Conciousness. The encounter between the Old and New World). Amsterdam 1986, éditions Ambo.
} 
sur la civilisation. Nous ne sommes qu'en partie les héritiers des Grecs et des Romains, plus proches finalement de ces «Sauvages américains » que nous ne le croyons. Dans cette confrontation tragique avec l'Autre, par laquelle l'Occident s'empare du Nouveau Monde, naît la conscience coupable que le "Sauvage» est humain comme nous et le soupçon inquiétant que nousmêmes avons été un jour, pas si lointain, des «Sauvages » comme eux. Aussi, dans cette rencontre avec l'Autre se constituent, en quelque sorte symétriquement, le projet d'une Anthropologie qui saisirait la différence et la proximité de l'Autre et, d'autre part, le projet d'une Préhistoire qui recueillerait la mémoire de nos origines «sauvages » d'avant la civilisation. L'une informant l'autre de ses découvertes; l'une comme l'autre tentant d'échapper au carcan des traditions de pensée établies - des a priori et du conformisme. C'est un rêve, bien sûr.

Les «Sauvages » nus des Amériques et les Européens qui tenaient leurs hautsde-chausse pour un signe distinctif de civilisation ${ }^{50}$ ont maintenant disparu depuis longtemps. Ceci est devenu de l'histoire, ou de l'archéologie. Mais il existe pourtant un passage par où aller à la rencontre de ce moment éblouissant où nous les avons connus pour la première fois, dans " cet échange où l'on se change sans pour autant se perdre ni se dénaturer", ce moment fragile et éphémère qui est " aussi une occasion de poésie, là où l'être dans le monde grandit l'être-en-soi », comme l'écrivent Édouard Glissant et Patrick Chamoiseau. ${ }^{51}$ L'archéologie montre inlassablement, partout dans le monde, qu'il n'a jamais été, à aucun moment de l'histoire de l'humanité, ni culture "pure», ni identité "en soi». Les vestiges des civilisations du passé ne révèlent qu'un enchevêtrement d'identités multiples, sans cesse recomposées, une intrication de strates culturelles incessamment recouvertes, continuellement remises en jeu. Nous n'existons que dans la relation; nous n'avons d'identité que dans l'interférence avec l'Autre, qui nous dérange et nous change. Comme l'écrivent encore Glissant et Chamoiseau :

Ainsi tout conquérant est-il secrètement conquis. Tout dominant s'abîme dans l'alchimie de sa domination même. Prendre ouvre des espaces à de toutes secrètes emprises. La force brutale et aveugle livre celui qui l'exerce à d'imparables faiblesses. En prenant le monde, l'Occident s'est aussi fait prendre par lui. ${ }^{52}$

\footnotetext{
50 «Tout cela ne va pas trop mal » concluait Montaigne à propos de la prétendue sauvagerie des Cannibales du Brésil «mais quoi, ils ne portent pas de hauts-de-chausses! » (Montaigne, op. cit., Livre Ier, chapitre 31 (des cannibales), p. 164.

51 Edouard Glissant et Patrick Chamoiseau, Quand les murs tombent. L'identité nationale hors-laloi ? Paris 2007, éditions Galaade, Institut du Tout-Monde, p. 9-10.

52 Glissant et Chamoiseau, op. cit., p. 14.
} 